\title{
First metabolic oncology inhibitor gets FDA green light, with record price tag
}

On August 1, the US Food and Drug Administration (FDA) granted full approval to an isocitrate dehydrogenase 2 (IDH2) enzyme inhibitor to treat relapsed or refractory acute myelogenous leukemia (AML). It's the first approved drug that targets cancer energy metabolism, a field resurrected 20 years ago and based on observations made by German biochemist Otto Warburg before the Second World War. Agios Pharmaceuticals in Cambridge, Massachusetts, discovered the oral compound, IDHIFA (enasidenib), which Celgene in Summit, New Jersey, co-developed. The FDA approval is for patients with tumor IDH2 gene mutations. "There is no doubt in my mind that this drug will be a standard-ofcare option," says hematologist and stem cell researcher Ravi Majeti at Stanford University in Palo Alto, California. "The only question is if it can be moved up front" as initial therapy. Trials addressing that are ongoing.

Agios alone is developing a second drug, ivosidenib, to treat IDH1-mutant AML, and will submit that FDA application by year's end, says company CEO David Schenkein. Together, IDH mutations are present in about $22 \%$ of the 21,000 new AML cases diagnosed in the US each year. Many solid tumors also harbor IDH mutations.

IDHIFA went from the first dosing in patients to FDA approval in less than four years, a "near record," says Cowen biotech analyst Eric Schmidt in New York. "It builds a lot of credibility for [Agios]." Schmidt forecasts annual IDHIFA sales of $\$ 585$ million by 2022 , and ivosidenib sales of $\$ 315$ million. Agios will earn about a $12.5 \%$ IDHIFA royalty from Celgene, estimates Goldman Sachs analyst Terence Flynn in New York.

IDHIFA's antitumor mechanism is unique. It doesn't kill leukemia cells, but causes them to differentiate, halting proliferation and clearing the bone marrow for normal immune cell production. In the phase $1 / 2$ trial that led to FDA approval, $40.3 \%$ of patients had an objective response, with a median response duration of 5.8 months (Blood 130, 722-731, 2017). 34 of 176 patients saw their leukemias eradicated, at least temporarily. This enabled some potentially curative bone marrow transplants, but IDHIFA itself "is likely not a curative therapy as monotherapy," notes Majeti. Side effects were generally mild, but there were some cases of differentiation syndrome, a spike in white blood counts with potentially severe fever, hypotension or pleural or pericardial effusion.

The clinical trial, though successful, did raise questions about IDHIFA's mechanism of action.

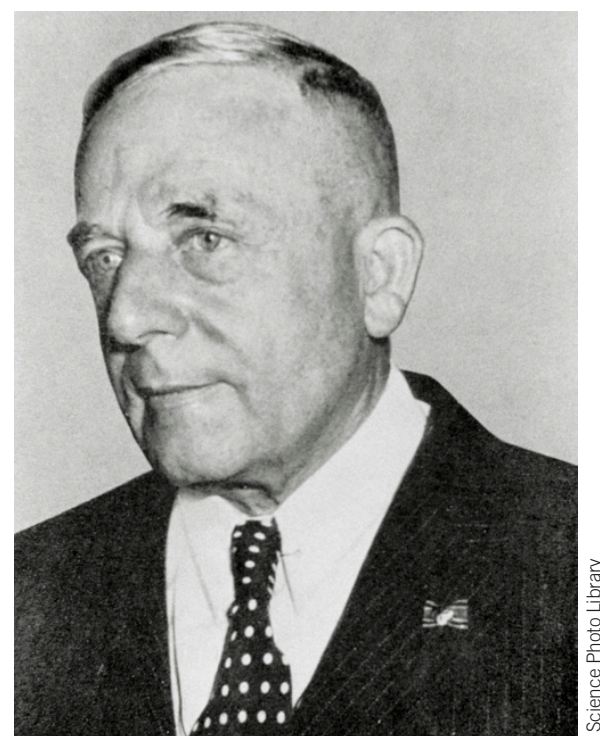

Otto Warburg, German biochemist, first observed that cancer cells metabolize glucose differently than normal cells. This so-called "Warburg effect" inspired today's cancer metabolism field, spearheaded by Agios Pharmaceuticals.

The drug's target, mutant IDH2, is a metabolic enzyme with epigenetic effects. It generates an oncometabolite, 2-hydroxyglutarate (2-HG), that indirectly affects the methylation state of DNA and prevents the expression of genes necessary for cell differentiation (Nat. Biotechnol. 28, 888-891, 2010). With the cells stuck in an undifferentiated state, they eventually become malignant.

In the trial, however, 2-HG suppression did not predict response (Blood 130, 732-741, 2017). "There's something more going on than just 2-HG” in IDH mutant cells, says Majeti.

The drug's mechanism of action is not fully understood, and it also remains unclear why some patients do not respond. "We've not been able to isolate a clear primary resistance mechanism," says Schenkein. In patients who respond but later relapse, there are cells with IDH2 mutations that survive. In complete responders, "the question is, are we eradicating [the mutation] from the stem cell [population]," says Schenkein. "It's way too early to know, but our suspicion is that the clone will come back if you're not continuing to suppress it."

Celgene has priced IDHIFA at just under $\$ 25,000$ per patient per month, the highest cancer drug price he's seen to date, says Schmidt, including for biologics. Schenkein says Agios had no input into the pricing decision.

Ken Garber Ann Arbor, Michigan 